\title{
Aportación de Teoría de la mente de la Batería NEPSY-II como medida de Coherencia central
}

\section{Contribution of the NEPSY-II Battery Theory of Mind subtest as a measure of central coherence}

\author{
Nerea Crespo-Eguílaz ${ }^{1}$ y Leyre Gambra Echeverría ${ }^{2}$ \\ ${ }^{1}$ Universidad de Navarra necrespo@unav.es \\ ${ }^{2}$ Universidad de Navarra lgambra@unav.es
}

Recibido: $11 / 9 / 2020$

Aceptado: 9/12/2020

\section{Copyright (C)}

Facultad de CC. de la Educación y Deporte. Universidad de Vigo

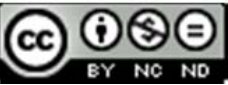

Dirección de contacto:

Nerea Crespo Eguílaz

Unidad de Neuropediatría del Dpto. de

Pediatría de la Clínica Universidad de Navarra

Avda. Pío XII, 36

31008 Pamplona

\begin{abstract}
Resumen
Introducción

El objetivo de esta investigación es analizar la utilidad clínica de "Teoría de la mente" (TM) de la NEPSY-II (Korkman y cols., 2014).

Metodología

Se aplica la TM a 244 escolares de 6 a 11 años con inteligencia normal: 194 del grupo control y 50 de los grupos clínicos: TEA-grado $1(\mathrm{n}=10)$; trastorno de aprendizaje procedimental (TAP, $n=24)$; y TDAH $(n=16)$.

Resultados y discusión

En los 21 ítems de la TM se realizan análisis factoriales exploratorio $(\mathrm{KMO}=0,62$ y MSA $=0,54-0,75)$ y confirmatorio $\left(\chi^{2}=22,75 p=0,504 ;\right.$ TLI $=1,04 ; C F I=1 ;$ RMSEA $\left.=0\right)$. Se obtienen 3 factores que explican el $62 \%$ de la varianza total: Teoría de la mente (ítems 1/2/7); Comprensión relatos sociales (ítems 5/8/10/14); y Coherencia contextual (items 9/12/16-21). Se calcula la validez y la fiabilidad y se aportan los estadísticos descriptivos.

En los dos primeros factores no se diferencian ninguno de los grupos (U-mannWhitney). En Coherencia contextual se diferencian el grupo TAP y los grupos control $(p=, 001)$ y TEA $(p=, 025)$. Está demostrado que las dificultades para comprender información rápida y simultánea e integrarla coherentemente forma parte del perfil neuropsicológico del TAP.

Conclusión

Esta nueva corrección e interpretación de la TM evalúa Coherencia central y la aportamos como uso adicional de la prueba.
\end{abstract}

\section{Palabras clave}

Coherencia Central, Teoría de la Mente, Trastorno de Aprendizaje Procedimental, Trastorno del Espectro del Autismo

\section{Abstract}

Introduction

The aim of this research is to analyze the clinical utility of the "Theory of Mind" (TM) subtest of NEPSY-II (Korkman et al., 2014).

Methodology 
TM was applied to 244 school aged children with normal intelligence: a control group $(\mathrm{n}=194)$ and clinical groups $(\mathrm{n}=50)$ : ASD-grade $1(\mathrm{n}=10)$; procedural learning disorder (PLD, $\mathrm{n}=24)$; and $\mathrm{ADHD}(\mathrm{n}=16)$.

Results and Discussion

We do a examination of the factorial structure through a exploratory $(\mathrm{KMO}=0.62$ and MSA $=0,54-0,75)$ and confirmatory $(\chi=22,75 \quad \mathrm{p}=0,504 ; \quad$ TLI $=1,04 ; \quad$ CFI $=1$; RMSEA $=0$ ) analyses. The 21 items of the TM. 3 factors are obtained that explain $62 \%$ of the total variance: Theory of mind (items 1/2/7); Social stories comprehension (items 5/8/10/14) and Contextual coherence (items 9/12/16-21). The descriptive statistics are provided from the calculation of validity and reliability.

None of the groups differ in the first two factors (U-mann-Whitney). In Contextual coherence, the PLD group and the control groups $(p=, 001)$ differed significantly of the ASD group $(p=, 025)$.

The difficulties in understanding information in a quick and simultaneous way and integrating it coherently have been shown to be part of the neuropsychological profile of the PLD.

Conclusión

Our study provide a new correction an interpretation of the TM that evalautes Central coherence.

\section{Key Words}

Central Coherence, Theory of Mind, Procedural Learning Disorder, Autism Spectrum Disorder

\section{INTRODUCCIÓN}

Las habilidades de teoría de la mente se refieren a la capacidad de atribuir estados mentales (sentimientos, creencias, intenciones y deseos) a los demás y a uno mismo. Se desarrollan tempranamente en el niño, comenzando con la capacidad para comprender las creencias de los demás acerca de una situación (teoría de la mente de primer orden), seguidas de la comprensión de que las personas tienen pensamientos o creencias sobre los pensamientos de otras personas (teoría de la mente de segundo orden) y finalmente se desarrollan habilidades mentalistas sociales más complejas (Brent et al., 2004). Baron-Cohen, Leslie y Frith (1985) encuentran que el $80 \%$ de una muestra de niños y adolescentes con autismo de alto funcionamiento presentan dificultades en tareas de falsa creencia.

La subprueba "Teoría de la mente" (TM) de la Batería neuropsicológica NEPSY-II (Korkman, Kirk y Kemp, 2014) examina la capacidad para comprender creencias, emociones e intenciones, y la conciencia de que otras personas tienen sus propios pensamientos y sentimientos. La prueba se divide en una tarea verbal que evalúa la capacidad de percibir el punto de vista de otra persona, y una tarea contextual que examina la capacidad de reconocer estados emocionales relacionados con diferentes contextos sociales.

En nuestra experiencia clínica en la consulta de Neuropediatría esta prueba parece no discriminar bien a los escolares con dificultades en el constructo neuropsicológico que da nombre a la misma. Por consiguiente, el objetivo de esta investigación es analizar su utilidad clínica en el diagnóstico de los trastornos del neurodesarrollo. 


\section{MÉTODO}

\subsection{Participantes}

La muestra está formada por 244 participantes que constituyen el grupo control de alumnos normotípicos $(n=194)$ y la muestra clínica $(n=50)$ de pacientes de nuestra Unidad de Neuropediatría afectos de: trastorno del espectro del autismo de grado 1 (TEA-1=10); trastorno de aprendizaje procedimental-no verbal (TAP=24); y trastorno por déficit de atención e hiperactividad (TDAH=16). Todos son escolares de Educación Primaria, de edades comprendidas entre 6 y 11 años, y con inteligencia normal, evaluada con el test de matrices progresivas de Raven (Raven, 2001).

En la Tabla 1 se especifican las frecuencias por sexo de toda la muestra y las medidas de tendencia central del Cociente intelectual de cada grupo clínico. No se dispone de este dato del grupo control, pero los criterios de exclusión de este grupo fueron presentar un bajo rendimiento académico, dificultades de aprendizaje o trastornos de conducta en el momento de la evaluación; objetivado por el profesor tutor correspondiente.

\begin{tabular}{|c|c|c|c|c|}
\hline & \multirow[t]{2}{*}{ Grupo control } & \multicolumn{3}{|c|}{ Muestra clínica } \\
\hline & & TAP & TDAH & TEA-1 \\
\hline $\mathrm{n}$ & 194 & 24 & 16 & 10 \\
\hline Varón & 76 & 22 & 14 & 8 \\
\hline Mujer & 118 & 2 & 2 & 2 \\
\hline CI: x (ds) & ---- & $100,4(9,98)$ & $104,8(8,65)$ & $\begin{array}{c}101,7 \\
(11,19)\end{array}$ \\
\hline CI: min-máx & --- & $82-129$ & $83-114$ & $83-119$ \\
\hline
\end{tabular}

Tabla 1. Frecuencias por sexo en la casuística y Estadísticos descriptivos del Cociente intelectual (CI) en la muestra clínica

\subsection{Instrumentos y procedimiento}

Se aplica la subprueba TM de la Batería neuropsicológica NEPSY-II (Korkman et al., 2014) a todos los participantes de esta investigación. Se divide en dos tareas verbal y contextual. En la tarea verbal, los participantes escuchan descripciones o ven imágenes de escenarios dónde se les hacen preguntas sobre el punto de vista de otra persona vista. Las preguntas miden diferentes aspectos como la comprensión de creencias, intenciones, pensamientos, ideas, comprensión del lenguaje figurado y habilidades de imitación. La tarea contextual consta de seis láminas de situaciones sociales cuya protagonista es una niña; cada una se acompaña de cuatro fotografías de la cara de dicha niña que expresan diferentes emociones, incluyendo la correspondiente al escenario representado. Está formada por 21 ítems que evalúan: la resolución de tareas de teoría de la mente de primer y segundo orden (ítems 1, 2, 6, 7, 8); la comprensión de verbos mentalistas (ítems 3, 5), de frases hechas de acuerdo al contexto (ítems 12, $13 \mathrm{y}$ 15) y de historias en las que hay que inferir intenciones (ítems 10 y 14); la imitación de gestos o la comprensión de la imitación de otro (ítems 4 y 9); la integración de las partes 
de un dibujo en un todo coherente (ítem 11); y, por último, la interpretación de expresiones faciales emocionales relacionadas con situaciones concretas (ítems del 16 al 21).

Además, se aplica a los 50 participantes de la muestra clínica la Prueba de Coherencia Central PCC (Gambra, 2020) y dos cuestionarios a sus padres: Children's Communication Checklist CCC (Bishop, 1998, 2003; Crespo-Eguílaz, Magallón, Sánchez-Carpintero y Narbona, 2016) y Childhood Autism Test CAST (Scott, BaronCohen, Bolton y Brayne, 2002), que se detallan a continuación:

- Prueba de Coherencia Central PCC (Gambra, 2020). Está formada por 10 subpruebas que se agrupan en cuatro dimensiones que miden el constructo neuropsicológico de Coherencia central: Inferencia, Detalle verbal, Simultaneidad y Contexto. En Inferencia se valora la comprensión de la ironía, de frases literales y de un relato no explícito. En Detalle verbal se evalúa la comprensión de preguntas y frases sintácticamente correctas pero absurdas por un detalle concreto. En Simultaneidad se trata de resolver tareas en las que la información que hay que tener en cuenta se presenta de forma simultánea. Y, por último, en Contexto se valora la comprensión de situaciones que requieren integrar la información del contexto en un todo coherente.

- Children's Communication Checklist CCC (Bishop, 1998, 2003). Es una prueba útil para la valoración de la pragmática lingüística y para la detección de posibles trastornos pragmáticos, como el trastorno de la comunicación social. La versión del CCC en castellano (Crespo-Eguílaz et al., 2016) consta de 55 ítems divididos en tres áreas de Pragmática, Relaciones sociales e Intereses. Dentro de la primera se detallan cinco dimensiones: habilidades de conversación; coherencia y comprensión; compenetración con el interlocutor; comunicación no verbal; y pertinencia de la conducta.

- Childhood Autism Spectrum Test CAST (Scott et al., 2002). Los padres contestan a 37 preguntas relacionadas con el uso del lenguaje, la interacción social e intersubjetividad, el tipo de intereses y actividades. Se utiliza como prueba de screening de trastornos del espectro del autismo de grado 1 (antes denominado síndrome de Asperger). Una puntuación significativa ( $\geq 15$ puntos de 31 puntos posibles) requiere indagar en la sintomatología conductual.

En una de nuestras investigaciones (Crespo-Eguílaz et al., 2020), analizamos el CAST para precisar diferentes dimensiones en su contenido y obtuvimos siete dimensiones pormenorizadas: Normas y turnos; Flexibilidad; Juego imaginativo; Percepción global; Pragmática; Intersubjetividad; e Interacción con iguales.

Con los datos obtenidos en el grupo control se realizan análisis factoriales exploratorio y confirmatorio, para agrupar los ítems de la prueba en dimensiones subyacentes. Con los datos obtenidos en los grupos clínicos se analiza la validez y la fiabilidad de las nuevas dimensiones, para aportar así una nueva versión de la prueba.

\section{RESULTADOS Y DISCUSIÓN}

\subsection{Subprueba TM original: rendimiento en los grupos clínicos}

En primer lugar, se compara el rendimiento obtenido en los grupos clínicos en la TM (Figura 1) y se comprueba que no hay diferencias significativas entre ellos (KruskalWallis: 8,474; sig ,076). 


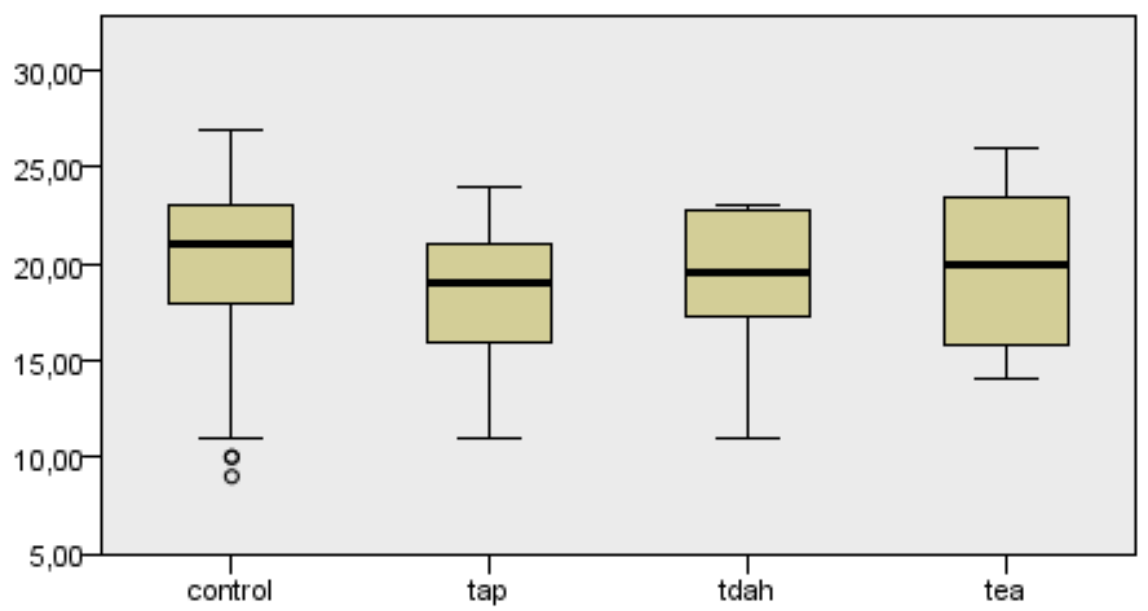

Figura 1. Rendimiento obtenido en la versión original de Teoría de la mente de la NEPSY II. Comparaciones entre grupos (Kruskal-Wallis de muestras independientes)

Sin embargo, en otros estudios con la subprueba TM de la NEPSY-II, obtienen un rendimiento estadísticamente menor el grupo con TEA, respecto al TDAH (Berenguer y Roselló, 2016); y el grupo con dislexia, respecto al grupo control (Cardillo, García, Mammarella y Cornoldi, 2018).

\subsection{Análisis factorial exploratorio y obtención de las dimensiones de la nueva versión de TM}

Se realiza un análisis factorial exploratorio de los 21 ítems de la prueba, en la mitad del grupo control ( $\mathrm{n}=97)$, con la intención de determinar el número y la naturaleza de los factores latentes que explican la variabilidad entre el conjunto de medidas observables. Se utiliza la técnica análisis factorial con los métodos Componentes principales y Rotación Varimax-Kaiser. Se adopta el criterio de la pendiente de Cattel para obtener los componentes. La medida de adecuación muestral de Kaiser-MeyerOlkin (KMO) y la medida de adecuación de la muestra para cada variable (MSA) son mayores de 0,5 $(\mathrm{KMO}=0,62$ y $\mathrm{MSA}=0,54-0,75)$, lo que indica una buena adecuación de los datos a un modelo de análisis factorial. Se obtienen tres factores, de acuerdo a las cargas factoriales y el contenido, que explican el $62 \%$ de la varianza total; todos los ítems con cargas factoriales $>0,41$ (ver Tabla 2).

Se establecen las siguientes denominaciones de las tres dimensiones obtenidas:

- 1. Teoría de la mente, 3 ítems: 1, 2 y 7

- 2. Comprensión de relatos sociales, 4 ítems: 5, 8, 10 y 14

- 3. Coherencia contextual, 8 ítems: $9,12,16-21$ 


\begin{tabular}{lccc}
\hline \multicolumn{4}{c}{ Factores o dimensiones } \\
\hline Ítems & 1 & 2 & 3 \\
\hline 1 &, 646 &,- 049 &, 455 \\
\hline 2 &, 828 &, 006 &, 096 \\
\hline 5 &,- 206 &, 542 &, 429 \\
\hline 7 &, 405 &, 317 &, 093 \\
\hline 8 &, 258 &, 786 &, 114 \\
\hline 9 &, 112 &, 088 &, 561 \\
\hline 10 y 14 &,- 036 &, 600 &, 342 \\
\hline 12 &, 099 &, 193 &, 570 \\
\hline $16-21$ &, 020 &,- 054 &, 787 \\
\hline
\end{tabular}

Tabla 2. Matriz de componentes rotados correspondiente al Análisis factorial exploratorio De los 21 ítems de la prueba, 6 ítems $(3,4,6,11,13,15)$ no se agrupan con ningún otro ítem, por lo que no configuran ningún factor. Los ítems 10 y 14 así como los ítems del 16 al 21 se analizan juntos puesto que forman parte de la misma tarea. Método de extracción: Análisis de componentes principales. Método de rotación: Normalización Varimax con Kaiser. En gris: variables que se agrupan en un factor.

\subsection{Análisis factorial confirmatorio de la nueva versión de TM}

El análisis factorial confirmatorio es una alternativa más exigente que el análisis factorial exploratorio que permite determinar a priori la estructura esperable (del conjunto total de las relaciones entre los elementos que lo configuran) de los datos a partir del modelo teórico ya existente y ponerla a prueba mediante técnicas estadísticas. Se utiliza el paquete estadístico IBM SPSS-AMOS graphics con el método de estimación de máxima verosimilitud. Para ello se utilizan los datos obtenidos en la segunda mitad del grupo control; los 97 escolares restantes.

Para la bondad de ajuste del modelo se emplean los indicadores sugeridos por $\mathrm{Hu}$ y Bentler (1995): el estadístico chi-cuadrado, la razón de chi-cuadrado sobre los grados de libertad (CMIND/DF), el índice de Tucker-Lewis (TLI), el índice de ajuste comparativo (CFI) y el error cuadrático medio de aproximación por grado de libertad (RMSEA). El estadístico Chi-cuadrado indica el ajuste absoluto del modelo pero, al ser muy sensible al tamaño de la muestra, se interpreta a su vez CMIND/DF; con valores inferiores a cuatro que indican un buen ajuste. Los índices TLI y CFI son superiores a 0,90 lo que sugiere un buen ajuste del modelo a los datos (Schermelleh-Engel, Moosbrugger y Müller, 2003). Por otro lado, el índice RMSEA es adecuado puesto que es menor que 0,05 (Browne y Cudeck, 1989).

En la Tabla 3 se representan los estadísticos empleados con los resultados de estimación de dichos parámetros junto con sus valores recomendados para la valoración de ajuste del modelo (Bryne, 2010; Kline, 2016). Como se puede observar, la muestra tiene un ajuste aceptable al modelo de tres factores.

\subsection{Nueva versión de TM: fiabilidad y validez}

Se calcula la fiabilidad de la TM con estas nuevas dimensiones (alfa de Cronbach: ,782) en toda la muestra estudiada.

Para llevar a cabo el estudio de la validez de la nueva versión de TM se realizan los análisis de correlación entre las tres dimensiones de la misma (Teoría de la mente, Comprensión de relatos sociales y Coherencia contextual) y el resto de las pruebas aplicadas. Se pretende comprobar si la prueba correlaciona con otras pruebas que miden 
dimensiones similares (validez convergente) y si no correlaciona con otras pruebas que miden dimensiones diferentes (validez discriminante).

\begin{tabular}{llcc}
\hline \multicolumn{1}{c}{ Estadísticos } & $\begin{array}{c}\text { Nivel de ajuste } \\
\text { recomendado }\end{array}$ & $\begin{array}{c}\text { Nivel de ajuste } \\
\text { obtenido }\end{array}$ \\
\hline Chi-cuadrado $\left(\chi^{2}\right)$ & & $p>, 05$ & $\begin{array}{c}\chi^{2}=22,75 \\
\mathrm{gl}=24 \\
p=, 504\end{array}$ \\
\hline Ajuste absoluto & $\mathrm{CMIN} / \mathrm{DF}$ & $<4$ & 0,95 \\
\hline $\begin{array}{l}\text { Ajuste comparativo o ajuste } \\
\text { incremental }\end{array}$ & TLI & & 1,04 \\
\hline Otros & CFI & $>, 95$ & 1 \\
\hline
\end{tabular}

Tabla 3. Resumen de los coeficientes e índices de bondad de ajuste del modelo propuesto CMIN/DF: $\chi^{2} / g 1$. NFI: Normed Fix Index - índice de ajuste no normalizado. GFI: Goodness-of-fit Index índice de bondad de ajuste. TLI: Tucker-Lewis Index. CFI: Comparative Fit Index - índice de ajuste comparativo. RMSEA: Mean Square Error of Approximation - raíz cuadrada del error cuadrático medio

Respecto a la Prueba de Coherencia Central PCC (Gambra, 2020), las dimensiones Teoría de la mente y Comprensión de relatos sociales de la TM correlacionan con las dimensiones Inferencia y Detalle verbal de la PCC. Estos resultados no sorprenden puesto que, para ponerse en el lugar del otro y para comprender historias sociales, hay que realizar inferencias y tener en cuenta los detalles verbales y extraverbales. Por otra parte, Coherencia contextual de la NEPSY-II correlaciona con las cuatro dimensiones de Coherencia central de la PCC (ver Tabla 4). Por consiguiente, estos resultados aportan la validez convergente de la dimensión Coherencia contextual de la nueva versión de TM como medida de Coherencia central.

\begin{tabular}{llccc}
\hline & & $\begin{array}{c}\text { Teoría de la } \\
\text { mente }\end{array}$ & $\begin{array}{c}\text { Comprensión de } \\
\text { relatos sociales }\end{array}$ & $\begin{array}{c}\text { Coherencia } \\
\text { contextual }\end{array}$ \\
\hline PCC & Inferencia &, $174^{*}$ &, $367 * *$ &, $344^{* *}$ \\
\hline & Detalle verbal &, $488^{* *}$ &, $302^{*}$ &, $276^{*}$ \\
\hline Simultaneidad &, 198 &, 214 &, $305^{*}$ \\
\hline CAST & Contexto &, 201 &, 222 &, $286^{*}$ \\
\hline & Pragmática &, 246 &,- 003 &,- 078 \\
\hline & Relación social &,- 039 &, 036 &, 077 \\
\hline & Intereses &,- 158 &,- 053 &,- 117 \\
\hline
\end{tabular}

** La correlación es significativa a nivel ,01 (bilateral). * La correlación es significativa a nivel ,05 (bilateral)

Tabla 4. Correlaciones entre la nueva versión de TM de la NEPSY-II y otras pruebas: la Prueba de Coherencia Central PCC y los cuestionarios Children's Communication Checklist CCC y Childhood Autism Test CAST 
Respecto al cuestionario Children's Communication Checklist CCC, la nueva versión de la TM no correlaciona con ninguna de las tres áreas del CCC ni con cada una de las cinco dimensiones que configuran el uso pragmático del lenguaje (ver Tabla 4).

En relación al Childhood Autism Spectrum Test CAST, la nueva versión de TM no correlaciona con la puntuación global del CAST (ver Tabla 4), ni con cada una de las siete dimensiones que la configuran.

Según los datos anteriormente expuestos, que indican ausencia de relación de la nueva versión de TM con pruebas que miden dimensiones diferentes, se aporta la validez discriminante de la nueva versión de TM.

\subsection{Nueva versión de TM: estadísticos descriptivos del grupo control}

En el grupo control se calculan los estadísticos descriptivos de las tres nuevas dimensiones (Teoría de la mente, Comprensión de relatos sociales y Coherencia contextual) y se detallan por sexo y en dos rangos de edad: de 6 a 8 años y de 9 a 11 años (Tabla 5).

\begin{tabular}{lccc}
\hline & $\begin{array}{c}\text { Teoría de la } \\
\text { mente }\end{array}$ & $\begin{array}{c}\text { Comprensión de } \\
\text { relatos sociales }\end{array}$ & $\begin{array}{c}\text { Coherencia } \\
\text { contextual }\end{array}$ \\
\hline 6-8 años $(\mathrm{n}=115): \mathrm{x}(\mathrm{s})$ & $3,17(1,05)$ & $3,34(1,78)$ & $6,76(1,66)$ \\
\hline 9-11 años $(\mathrm{n}=79): \mathrm{x}(\mathrm{s})$ & $3,65(0,74)$ & $4,32(1,42)$ & $7,57(1,21)$ \\
\hline$t$ Student: $\mathrm{F}(\mathrm{sig})$ & $8,806(, 002)$ & $1,585(, 000)$ & $8,458(, 000)$ \\
\hline niños $(\mathrm{n}=76): \mathrm{x}(\mathrm{s})$ & $3,32(1,01)$ & $3,63(1,78)$ & $7,01(1,49)$ \\
\hline niñas $(\mathrm{n}=118): \mathrm{x}(\mathrm{s})$ & $3,37(0,93)$ & $3,82(1,66)$ & $7,15(1,58)$ \\
\hline$t$ Student $: F($ sig) & $0,304(, 687)$ & $1,569(, 453)$ & $0,132(, 562)$ \\
\hline \multicolumn{1}{c}{ Sombreado en gris, diferencias significativas } & &
\end{tabular}

Tabla 5. Estadísticos descriptivos de la nueva versión de TM en el grupo control, por edad y por sexo

\subsection{Nueva versión de TM: estudio comparativo según la edad y el sexo}

Tras realizar el análisis de diferencia de medias t de Student, como puede apreciarse en la Tabla 6, se obtienen diferencias significativas en las tres nuevas dimensiones entre los dos grupos de edad (hasta 8 años y mayores de 8 años). Por consiguiente, hay una tendencia evolutiva en el desarrollo de estas habilidades durante la Educación Primaria. Sin embargo, no se encuentran diferencias significativas en cuanto al sexo en ninguna de las dimensiones.

\subsection{Nueva versión de TM: rendimiento en los grupos clínicos}

Con los baremos de referencia del grupo control aportados, teniendo en cuenta la edad, se tipifican las puntuaciones obtenidas de cada uno de los pacientes de la muestra clínica. Se comparan las medias entre todos los grupos en las tres dimensiones de la prueba (Kruskal-Wallis). En los escolares de 6 a 8 años no se obtienen diferencias significativas en el rendimiento obtenido en la nueva versión de TM. Sin embargo, en los participantes de entre 9 y 11 años se encuentran diferencias significativas entre el 
grupo TAP y los grupos control y TEA-1 (Tabla 6 y Figura 2); siendo el rendimiento menor en el grupo afecto de trastorno de aprendizaje procedimental.

\begin{tabular}{lccccc}
\hline & $\begin{array}{c}\text { Kruskal- } \\
\text { Wallis }\left(\mathrm{X}^{2}\right)\end{array}$ & sig. & $\begin{array}{c}\text { Diferencias entre } \\
\text { grupos }\end{array}$ & $\begin{array}{c}\text { U Mann } \\
\text { Whitney }\end{array}$ & sig. \\
\hline Teoría de la mente & 1,804 &, 614 & $\mathrm{~ns}$ & --- & --- \\
\hline Comprensión de relatos sociales & 3,13 &, 372 & $\mathrm{~ns}$ & --- & --- \\
\hline Coherencia contextual & 14,54 &, $002^{*}$ & TAP $<$ control & 30,65 &, $002^{*}$ \\
\hline
\end{tabular}

ns: no diferencia significativas *diferencias significativas $\mathrm{p}<, 05$

Tabla 6. Comparación del rendimiento en la nueva versión de la TM entre los grupos, en el rango de edad de 9-11 años

Los niños con trastorno de aprendizaje procedimental presentan una Coherencia central débil puesto que tienen dificultades para percatarse rápidamente de la coherencia o incoherencia de un material complejo y para describirlo verbalmente. Pueden perderse entre los detalles, en lugar de procesar la información de manera integral o correcta (Doty, 2019). Las dificultades específicas para la comprensión rápida, simultánea y coherente de imágenes o escenas complejas forman parte del perfil neuropsicológico del TAP (Crespo-Eguílaz, Narbona y Magallón, 2012; Crespo-Eguílaz y Narbona, 2009).

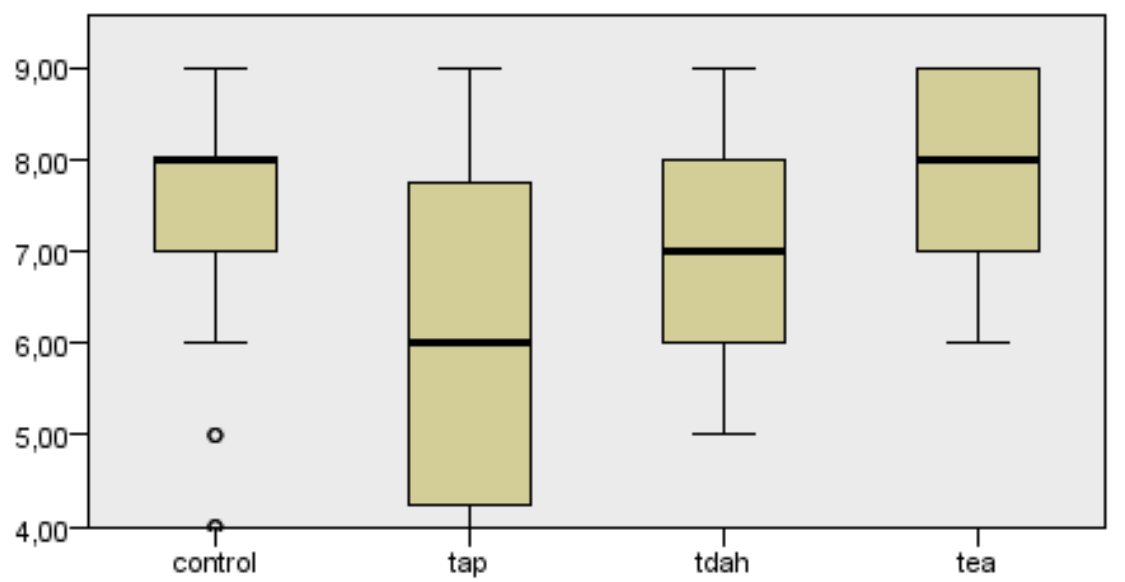

Figura 2. Rendimiento obtenido en la dimensión Coherencia contextual de la nueva versión de TM. Comparaciones entre grupos (Kruskal-Wallis de muestras independientes)

Está demostrado que los niños con TAP tienen dificultades en el reconocimiento de los detalles, el procesamiento simultáneo, la integración de las partes en el todo y la organización visuoespacial (Drummond, Ahmad y Rourke, 2005). Semrud-Clikeman, Walkowiak, Wilkinson y Christopher (2010) informan de hallazgos similares en comparación con otros grupos de niños con síndrome de Asperger y TDAH. Los escolares con TAP manifiestan un déficit para la comprensión de la información extraverbal (expresiones faciales, gestos, mímica, actitudes corporales, inflexiones prosódicas y el resto del entorno visual) así como una dificultad para integrar y comprender dicha información del contexto (Mammarella, Coltri, Lucangeli y Cornoldi, 2009). Por tanto, les cuesta entender el lenguaje figurativo, las ironías y las bromas; pueden interpretar el lenguaje de forma literal; su comunicación no es efectiva en los contextos cotidianos; y tienen problemas para adaptarse a situaciones novedosas de 
interacción social (Cornoldi, Fine y Mammarella, 2016; Crespo-Eguílaz y Narbona, 2009; Sans, Colomé, López-Sala y Boix, 2009).

En el TEA la teoría de la Coherencia central es respaldada inicialmente por estudios que afirman que en las pruebas de preferencia local su desempeño es significativamente mejor que aquellos con desarrollo normotípico (Jolliffe y Baron-Cohen, 1997) y que muestran un bajo rendimiento en las pruebas de preferencia global (Rinehart et al., 2000). Más tarde, una versión modificada de la teoría de la Coherencia central afirma que el sesgo de procesamiento local en individuos con TEA puede superarse en tareas con demandas explícitas de procesamiento global (Happé y Frith, 2006), aunque les resulta difícil el procesamiento global cuando se trata de partes incongruentes más pequeñas (Mottron et al., 2006). Por otra parte, este estilo de procesamiento de la información no es una característica universal en todo el espectro del autismo (Happé y Frith, 2006). Quizás por este motivo, los escolares con TEA1 de nuestra casuística no destacan por un bajo rendimiento en Coherencia central.

\section{CONCLUSIONES}

Los resultados de este estudio corroboran que esta nueva corrección e interpretación de la TM evalúa el constructo neuropsicológico de Coherencia central. Concretamente la dimensión Coherencia contextual nos permite diferenciar a los escolares con Coherencia central débil como los pacientes con trastorno de aprendizaje procedimental. Esta nueva versión de TM aporta información complementaria útil en el diagnóstico diferencial de patologías del neurodesarrollo que comparten algunos síntomas; puede ayudar a orientar la intervención psicoeducativa de estos escolares $y$, por tanto, a mejorar las habilidades afectadas.

\section{BIBLIOGRAFÍA}

Baron-Cohen, S., Leslie, A. M. y Frith, U. (1985). Does the autistic child have theory of mind? Cognition, 21, 37-46.

Berenguer, C. y Roselló, B. (2016). El valor de la Teoría de la Mente en la diferenciación de niños con TDAH y con TEA. En J.L. Castejón (Ed.), Psicología y educación: Presente y futuro (pp. 690-699). Madrid, ACIPE-Asociación Científica de Piscología y Educación

Bishop, D.V.M. (1998). Development of the Children's Communication Checklist (CCC): A method for assessing qualitative impairment in children. Journal of Child Psychology and Psychiatry and allied disciplines, 39(6), 879-891.

Bishop, D.V.M. (2003). The Children's Communication Checklist second edition (CCC- 2). Londres: The Psychological Corporation.

Brent, E., Rios, P., Happé, F. y Charman, T. (2004). Performance of children with autism spectrum disorder on advanced theory of mind tasks. Autism, 8, 283-299.

Browne, M.W. y Cudeck, R. (1989). Single sample cross-validation indices for covariance structures. Multivariate Behavioral Research, 24(4), 445-455.

Bryne, B.M. (2010). Structural equation modeling with Amos. Basic concepts, applications and programming (2a ed.). Nueva York: Routlege-Taylor and Francis Group.

Cardillo, R., Garcia, R.B., Mammarella, I.C. y Cornoldi, C. (2018). Pragmatics of language and theory of mind in children with dyslexia with associated language difficulties or nonverbal learning disabilities. Applied Neuropsychology: Child, 7(3), 245-256. 
Cornoldi, C., Fine, J. y Mammarella, I. C. (2016). Nonverbal learning disability. New York, NY: Guilford Press.

Crespo-Eguílaz, N., Gambra, L., Miguéliz, M., Luna, A., Ruiz, J., Abete, A., Ramos, E., Vergara, M. y Reyes, C. (22-24 de abril de 2020-aplazado). El análisis pormenorizado del Chilhood asperger síndrome test permite diferenciar subtipos clínicos. Comunicación oral. 8th International congress of educational sciences and development. Palacio de Congresos de Pontevedra, España.

Crespo-Eguílaz, N., Magallón, S., Sánchez-Carpintero, R. y Narbona, J. (2016). La adaptación al castellano de la Children's Communication Checklist permite detectar las dificultades en el uso pragmático del lenguaje y diferenciar subtipos clínicos. Revista de Neurología, 62(Supl 1), 49-57.

Crespo-Eguílaz, N. y Narbona, J. (2009). Trastorno de aprendizaje procedimental: características neuropsicológicas. Revista de Neurología, 49(8), 409-416.

Crespo-Eguílaz, N., Narbona, J. y Magallón, S. (2012). Disfunción de la coherencia central en niños con trastorno de aprendizaje procedimental. Revista de Neurología, 55(9), 513-519.

Doty, N. (2019). Nonverbal Learning Disability. En: H. Wilson y E. Braaten (Eds.), The Massachusetts General Hospital Guide to Learning Disabilities. Current Clinical Psychiatry. Boston: Humana Press.

Drummond, C.R., Ahmad, S.A. y Rourke, B.P. (2005). Rules for the classification of younger children with nonverbal learning disabilities and basic phonological processing disabilities. Archives of Clinical Neuropsychology, 20(2), 171-182.

Gambra, L. (2020). Coherencia central: diseño y validación de una prueba de evaluación y estudio en el trastorno de aprendizaje procedimental (Tesis doctoral). Pamplona: Universidad de Navarra.

Happé, F. y Frith, U. (2006). The weak coherence account: detail-focused cognitive style in autism spectrum disorders. Journal of Autism and Developmental Disorders, 36(1), 5-25.

Hu, L. y Bentler, P. (1995). Evaluating model fit. En R. Hoyle (Ed.), Structural equation modelling: Concepts, issues and applications (pp. 76-99). Thousand Oaks: Sage Publications.

Jolliffe, T. y Baron-Cohen, S. (1997). Are people with autism and Asperger syndrome faster than normal on the Embedded Figures Test? Journal of Child Psychology and Psychiatry, 38(5), 527-534.

Kline, R.B. (2016). Principles and practice of structural equation modeling (4.a ed.). Nueva York: The Guilford Press.

Korkman, M., Kirk, U. y Kemp, S. (2014). NEPSY-II: Manual clínico y de interpretación. España: Pearson, The Psychological Corporation.

Mammarella, I.C., Coltri, S., Lucangeli, D. y Cornoldi, C. (2009). Impairment of simultaneousspatial working memory in nonverbal (visuospatial) learning disability: A treatment case study. Neuropsychological Rehabilitation, 19(5), 761-780.

Mottron, L., Dawson, M., Soulieres, I., Hubert, B. y Burack, J.A. (2006). Enhanced perceptual functioning in autism: An update, and eight principles of autistic perception. Journal of Autism and Developmental Disorders, 36(1), 27-43.

Raven, J.C. (2001). Test de matrices progresivas. Madrid: TEA Ediciones.

Rinehart, N.J., Bradshaw, J.L., Moss, S.A., Brereton, A.V. y Tonge, B.J. (2000). Atypical interference of local detail on global processing in high-functioning autism and Asperger's disorder. The Journal of Child Psychology and Psychiatry and Allied Disciplines, 41(6), 769-778.

Sans, A., Colomé, R., López-Sala, A. y Boix, C. (2009). Trastorno de aprendizaje no verbal: características cognitivo-conductuales $\mathrm{y}$ aspectos neuropsicológicos. Revista de Neurología, 48, 77-81. 
Schermelleh-Engel, K., Moosbrugger, H. y Müller, H. (2003). Evaluating the fit of structural equation models: Tests of significance and descriptive goodness-of-fit measures. Methods of Psychological Research Online, 8(2), 23-74.

Scott, F.J., Baron-Cohen, S., Bolton, P. y Brayne, C. (2002). The CAST (Childhood Asperger Syndrome Test) Preliminary development of a UK screen for mainstream primary-schoolage children. Autism, 6(1), 9-31.

Semrud-Clikeman, M., Walkowiak, J., Wilkinson, A. y Christopher, G. (2010). Neuropsychological differences among children with Asperger syndrome, nonverbal learning disabilities, attention deficit disorder, and controls. Developmental Neuropsychology, 35(5), 582-600. 